NASA Contractor Report 191452

ICASE Report No. 93-18

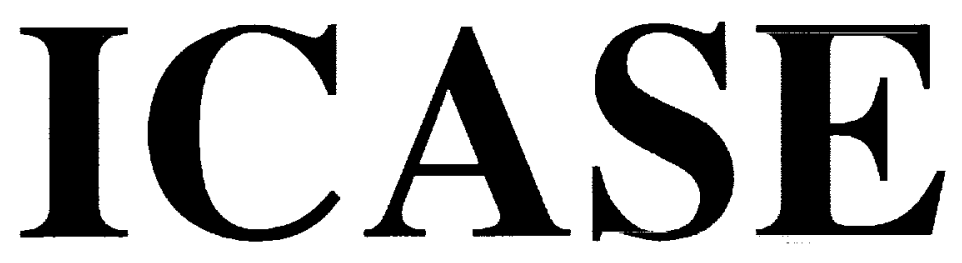

\title{
AN EFFICIENT MODEL FOR COUPLING STRUCTURAL VIBRATIONS WITH ACOUSTIC RADIATION
}

\section{Abdelkader Frendi \\ Lucio Maestrello \\ Lu Ting}

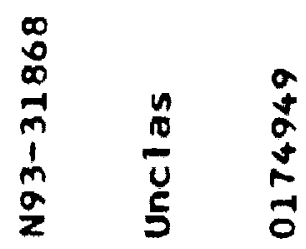

NASA Contract Nos. NAS1-19480 and NAS 1-18605 April 1993

Institute for Computer Applications in Science and Engineering NASA Langley Research Center Hampton, Virginia 23681-0001

Operated by the Universities Space Research Association

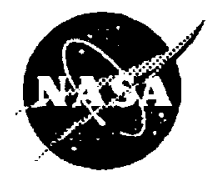

National Aeronautics and Space Administration Langley Research Center Hampton, Virginia 23681-0001

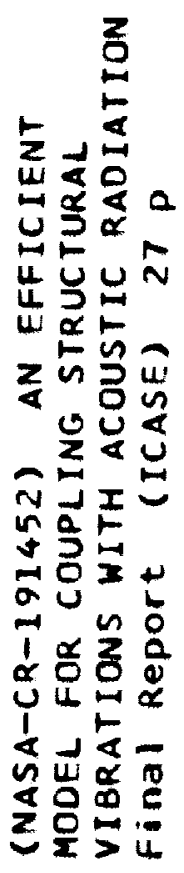




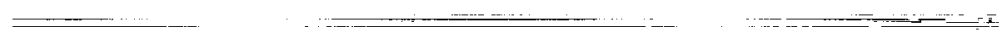




\title{
AN EFFICIENT MODEL FOR COUPLING STRUCTURAL VIBRATIONS WITH ACOUSTIC RADIATION
}

\author{
Abdelkader Frendi \\ Analytical Services and Materials, Inc. \\ Hampton, VA 23666 \\ Lucio Maestrello \\ NASA Langley Research Center \\ Hampton, VA 23681-0001 \\ Lu Ting ${ }^{1}$ \\ Courant Institute of Mathematical Sciences \\ New York, NY 10012
}

\begin{abstract}
We study the scattering of an incident wave by a flexible panel. The panel vibration is governed by the nonlinear plate equations while the loading on the panel, which is the pressure difference across the panel, depends on the reflected and transmitted waves. Two models are used to calculate this structural-acoustic interaction problem. One solves the three dimensional nonlinear Euler equations for the flow-field coupled with the plate equations (the fully coupled model). The second uses the linear wave equation for the acoustic field and expresses the load as a double integral involving the panel oscillation (the decoupled model). The panel oscillation governed by a system of integro-differential equations is solved numerically and the acoustic field is then defined by an explicit formula. Numerical results are obtained using the two models for linear and nonlinear panel vibrations. The predictions given by these two models are in good agreement but the computational time needed for the "fully coupled model" is 60 times longer than that for "the decoupled model".
\end{abstract}

\footnotetext{
${ }^{1}$ This research was supported by the National Aeronautics and Space Administration under NASA Contract Nos. NAS1-19480 and NAS1-18605 while the third author was in residence at the Institute for Computer Applications in Science and Engineering (ICASE), NASA Langley Research Center, Hampton, VA 2.3681-0001.
} 
$\ldots$

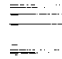

.

=

छ-

$=$ 


\section{Introduction}

We consider the scattering of an incident acoustic wave by a planar interface, the ( $x$, $z$ ) plane. The interface is a rigid surface except on the flexible thin panel, $\mathcal{D}$, with panel thickness $h$ much smaller than the size $L$ of the panel, or the size of $\mathcal{D}$,

$$
h / L \ll 1 .
$$

This is a basic structural-acoustic interaction problem simulating the transmission of an external acoustic source, say the engine noise, through the airframe into its interior. It is an interaction problem because the incident wave excites panel oscillation which in turn induces transmitted and diffracted waves. Meanwhile, these waves contribute to the loading on the panel. Although in an engineering problem, the typical amplitude of the transverse displacement, $\eta^{*}$, has to be much smaller than the size of the panel, i. e.,

$$
\eta^{*} / L \ll 1
$$

the problem can be nonlinear in three different aspects:

(1) The panel oscillation can be nonlinear when the transverse displacement of the panel is not much smaller than the panel thickness $h$, that is

$$
\eta^{*} \approx h .
$$

Nonlinear plate equations are needed usually when the incident wave is in resonance with the panel oscillation.

(2) In the near-field, of length scale $L$, the flow-field is linear and obeys the simple wave equation but in the far-field, of length scale much larger than $L$, the flow-field may become nonlinear when the second order terms are needed to account for the gradual steepening of compression waves.

(3) In case the flow-field is nonuniform and/or the initial pressure variation is no longer much smaller than the ambient pressure then we have nonlinear flow in the near-field. 
We have to solve the Euler equations with appropriate initial data to simulate an incident wave and far-field conditions.

Regardless of whether the flow-field is linear or nonlinear, the plate should have sufficient rigidity so that the transverse displacement of the plate remains small and hence the governing equations for the plate derived under assumption (1.1) remain valid.

For the problem of sound transmission through a moving airframe, the external acoustic field is governed by the convective wave equation while that in the interior by the simple wave equation. Also the pressure and density in the exterior can be much lower than those in the interior. In the simple model simulating the experimental data with no-flow on both sides of the panel, both acoustic fields are governed by the simple wave equation with the same ambient pressure and density. The validity of the linear theory for the flow-field is confirmed by the investigations of Frendi et al. [1,2] and Maestrello et al. [3]. They showed that even when the panel vibration is nonlinear, the acoustic field can remain linear.

The pressure difference across the panel induces the panel oscillation which in turn excites the transmitted wave and an additional reflected wave. Thus the solution of the panel oscillation is coupled with the solutions of the scattered and transmitted waves which satisfy the far-field radiation conditions for outgoing plane waves (see for example [4]). In general numerical solution of the scattering of an incident wave by an elastic scatterer, an interface, or a panel, requires the introduction of a finite computational domain for both acoustic fields. Higher order radiation conditions were derived [5-6] so that they can be imposed on the boundary of the finite computational domain to give more accurate approximation to the solution in the unbounded domain. Since the size of the computational domain has to be much larger than the size of the panel or a scatterer, numerical solution of this three-dimensional unsteady problem is very tedious especially when the computation has to be continued for a long time relative to the period of oscillation of the panel. Furthermore, the accuracy of the solution depends not only on that of the numerical solution of the differential equations but also on the approximate boundary conditions. Refinements of the grid size and time step have to go with an enlargement of the computational domain. It is desirable to find exact boundary conditions so that the computational 
domain does not have to be much larger than the scatterer and is independent of the choice of grid size. The exact conditions were presented in [7] for a scatterer or inhomogeneous medium of bounded support. With the scatterer inside the computational domain, the integral representation of the solution of the simple wave equation is applicable to the region outside and on the boundary of the computational domain.

Here the scatterer is a panel embedded in a rigid plane. The integral representation can be applied on the panel and the integrand involves only the normal velocity of the panel $[8,9]$. Thus we have a system of integro-differential equations for the panel oscillation decoupled from the acoustic field. This system is referred to as the decoupled model.

For the case that the acoustic wave length is much smaller than $L$, the integral representation can be approximated by the local derivatives of the panel oscillation and hence the integro-differential equations are reduced to partial differential equations $[8,9]$. This approximation is not applicable here because in the experimental data, the acoustic wave length is comparable to $L$. We need numerical solution of the integro-differential equations for the panel oscillation and then we can use the integral representation to evaluate the acoustic field and the nonlinear waves in the far-field by Whitham's theory or matched asymptotics.

Note that the solution of the decoupled panel oscillation is an unsteady two dimensional problem in the finite domain $\mathcal{D}$ and is several orders of magnitude simpler than the solution of the fully coupled problem. To show the efficiency of the decoupled model and the accuracy of the solution even when the panel oscillation is nonlinear, we compare the solution of this decoupled system with the solution of the fully coupled problem for which we solve the three-dimensional nonlinear Euler equations for the flow-field coupled with the nonlinear equations for the panel oscillations.

In the next section we present the mathematical formulations of these two models and discuss their applications in general. Section 3 describes the various numerical techniques used to solve the problems involved. The results and discussion are given in section 4 , and the conclusions are in section 5 . 


\section{Formulation of the Two Models}

The physical problem being studied experimentally is that of the nonlinear oscillations of a typical aircraft panel excited by harmonic plane waves at normal incidence. The panel is clamped onto a large rigid plate. Let the typical panel be represented by a rectangular domain $\mathcal{D}$ in the $(x, z)$ plane, with length $L$, width $W$ and one vertex located at $\left(x_{0}, z_{0}\right)$, i. e.,

$$
\mathcal{D}=\left\{(x, z) \mid x_{0}<x<x_{0}+L, z_{0}<z<z_{0}+W\right\} .
$$

The compliment of $\mathcal{D}$ in the $(x, z)$ plane represents the rigid plate. The incident side is the half space $y>0$ and the transmitted side is the half space $y<0$. Both sides have the same ambient pressure $p_{\infty}$ and temperature $T_{\infty}$ and hence the same speed of sound $C$ and density $\rho_{\infty}$. We denote the pressure and velocity potential of the flow-field by $p$ and $\phi$ and use the superscripts + and - to denote the quantities on the sides $y>0$ and $y<0$. On the side $y>0$, the superscripts $i$ and $r$ are used to denote the quantities associated with the incident and reflected waves, respectively. These two waves are mirror images with respect to the $(x, z)$ plane. When the incident waves are plane waves advancing in the direction opposite to the $y$ axis and hitting the $(x, z)$ plane at $t=0$, we have

$$
\phi^{(i)}(t, x, y, z)=f(C t+y) \quad \text { and } \quad \phi^{(r)}(t, x, y, z)=f(C t-y)
$$

with $f(\xi)=0$ for $\xi \leq 0$. Therefore $\phi^{(r)}=0$ when $0 \geq y<C t$.

Transverse oscillation, $\eta$, of the rectangular flexible panel, $\mathcal{D}$, is excited by the pressure difference across the panel for $t>0$ and the oscillation in turn induces scattered waves $\phi^{s}$ in the incident side and transmitted waves $\phi^{t}$. Both waves have the homogeneous initial data at $t=0$,

$$
\begin{array}{ll}
\phi^{(s)}=\phi_{t}^{(s)}=0, & y>0, \\
\phi^{(t)}=\phi_{t}^{(t)}=0, & y<0 .
\end{array}
$$

Under linear theory, we have

$$
\begin{aligned}
\phi^{(r)}(t, x, y, z) & =\phi^{(i)}(t, x,-y, z) \\
\phi^{(t)}(t, x,-y, z) & =-\phi^{(s)}(t, x, y, z) \text { for } y>0 .
\end{aligned}
$$


The wave fronts of $\phi^{(s)}$ and $\phi^{(t)}$ are the envelopes of the sonic spheres with radius $C t$ and centered on the panel in $y>0$ and $y<0$, respectively. The pressure in the incident and transmitted sides are

$$
\begin{array}{lrl}
p^{+}-p_{\infty}=p^{(i)}+p^{(r)}+p^{(s)}, & & y>0 \\
p^{-}-p_{\infty}=p^{(t)}, & & y<0
\end{array}
$$

and the pressure difference across the panel is

$$
\triangle p=p^{-}-p^{+}=2 p^{(t)}-\left[p^{(i)}+p^{(r)}\right], \quad y=0, \quad(x, z) \in \mathcal{D} .
$$

In this section we formulate two mathematical models to compare with and compliment the experimental studies. We present the system of equations governing the nonlinear flow-field and panel oscillation (the fully coupled model) in Section 2.1 and formulate the system of integro-differential equations for nonlinear panel oscillation including the effect of a linear acoustic field (the decoupled model) in Section 2.2 .

\subsection{The Fully Coupled Model}

In this model the structural-acoustic interaction is analyzed by solving the threedimensional nonlinear Euler equations together with the nonlinear plate equations. The configuration of the computational domain is shown in Fig. 1. In cartesian coordinates, $x$, $y$ and $z$, the compressible, nonlinear Euler equations can be written in conservation form as

$$
Q_{t}=F_{x}+G_{y}+H_{z}
$$

where $Q$ is the vector $(\rho, \rho u, \rho v, \rho w, e), \rho$ is the density, $\rho u, \rho v$ and $\rho w$ are the $x, y$ and $z$ momenta respectively, and $e$ is the total energy per unit volume given by

$$
e=\frac{1}{2} \rho\left(u^{2}+v^{2}+w^{2}\right)+\rho c_{v} T
$$

with $c_{v}$ being the specific heat at constant volume. In eq. (2.7), the functions $F, G$ and 
$H$ are:

$$
\begin{aligned}
& F=\left(\begin{array}{c}
\rho u \\
\rho u^{2}+p \\
\rho u v \\
\rho u w \\
u(e+p)
\end{array}\right), \\
& G=\left(\begin{array}{c}
\rho v \\
\rho u v \\
\rho v^{2}+p \\
\rho v w \\
v(e+p)
\end{array}\right)
\end{aligned}
$$

and

$$
H=\left(\begin{array}{c}
\rho w \\
\rho u w \\
\rho v w \\
\rho w^{2}+p \\
w(e+p)
\end{array}\right)
$$

In addition to eq. (2.7), the equation of state of an ideal gas is used:

$$
p=\rho R T,
$$

where $p$ is the pressure, $R$ the gas constant, and $T$ the temperature. Since we assume that the incident wave hits the panel at $t=0$, the flow on the transmitted side, $y<0$, is at rest for $t \leq 0$. The initial data at $t=0$ for $y<0$ are

$$
u=v=w=0, p=p_{\infty} \quad \text { and } \rho=\rho_{\infty} .
$$

On the rigid plate we have zero normal velocity,

$$
v(x, 0, z)=0 \quad \text { for } \quad(x, z) \notin \mathcal{D}
$$

The motion of the flexible panel, $\mathcal{D}$, is described by a system of three, nonlinear partial differential equations given by [10];

$$
\begin{aligned}
D \nabla^{4} \eta+\rho_{p} h \eta_{t t}+\gamma \eta_{t} & =\Delta p \\
& +\frac{E h}{1-\nu^{2}}\left[\left(u_{x}^{0}+\frac{1}{2} \eta_{x}^{2}\right)\left(\eta_{x x}+\nu \eta_{z z}\right)\right. \\
& +\left(w_{z}^{0}+\frac{1}{2} \eta_{z}^{2}\right)\left(\eta_{z z}+\nu \eta_{x x}\right) \\
& \left.+(1-\nu) \eta_{x z}\left(u_{z}^{0}+w_{x}^{0}+\eta_{x} \eta_{z}\right)\right]
\end{aligned}
$$




$$
\begin{aligned}
& u_{x x}^{0}+d_{1} u_{z z}^{0}+d_{2} w_{x z}^{0}=-\eta_{x}\left(\eta_{x x}+d_{1} \eta_{z z}\right)-d_{2} \eta_{z} \eta_{x z} \\
& w_{z z}^{0}+d_{1} w_{x x}^{0}+d_{2} u_{x z}^{0}=-\eta_{z}\left(\eta_{z z}+d_{1} \eta_{x x}\right)-d_{2} \eta_{x} \eta_{x z}
\end{aligned}
$$

where

$$
\begin{gathered}
\nabla^{4} \eta=\eta_{x x x x}+2 \eta_{x x z z}+\eta_{z z z z} \\
d_{1}=\frac{1-\nu}{2}, \quad d_{2}=\frac{1+\nu}{2}, \quad D=\frac{E h^{3}}{12\left(1-\nu^{2}\right)},
\end{gathered}
$$

$u^{0}$ and $w^{0}$ are the in-plane displacements, and $\eta$ is the transverse displacement. The physical constants for the panel appearing in (2.13) are; the stiffness $(D)$, the density $\left(\rho_{p}\right)$, the thickness $(h)$, the physical damping $(\gamma)$, the modulus of elasticity $(E)$ and the Poisson ratio ( $\nu)$. The system of equations (2.13) is solved subject to the homogeneons initial condition

$$
\text { at } t=0 \quad u^{0}=w^{0}=\eta=\eta_{t}=0
$$

and the clamped boundary conditions on $\mathcal{C}$ given by

$$
\begin{array}{ll}
x=x_{0}, x_{0}+L, & u^{0}=w^{0}=\eta=\eta_{x}=0, \\
z=z_{0}, z_{0}+W, & u^{0}=w^{0}=\eta=\eta_{z}=0 .
\end{array}
$$

In eq. (2.13), the load $\Delta p$ defined by (2.6) contains the coupling with the acoustic field $p^{t}$ and the forcing term $\left(p^{(i)}+p^{(r)}\right)$ which represents the load on the panel if the panel were rigid. We assume the forcing term to be a harmonic wave of the form

$$
p^{(i)}+p^{(r)}=\epsilon \sin (\omega t) H(t) \quad \text { on } \quad y=0^{+}
$$

where $\epsilon$ and $\omega$ are the amplitude and frequency of the wave and $H$ denotes the Heaviside unit step function. The load on the panel $\mathcal{D}$ given by $(2.6)$ becomes

$$
\Delta p=\left[2 p^{l}-\epsilon \sin (\omega t)\right] H(t)
$$

Another condition coupling the flow-fields and the panel oscillation is the kinematic conditions,

$$
\eta_{t}(t, x, z)=v^{ \pm}\left(t, x, 0^{ \pm}, z\right), \quad(x, z) \in \mathcal{D}
$$


They are imposed on $y=0^{ \pm}$because of the small panel displacement, (1.1). The numerical scheme for the solution of the nonlinear plate equation (2.13) and the Euler equations (2.7) for the transmitted waves with homogeneous initial data will be described in Sec. 3 .

\subsection{The Decoupled Model}

Now we analyse the nonlinear panel oscillation excited by a weak incident pressure wave, $p^{(i)}$, under the assumption that the pressure fluctuation remains much smaller than the ambient pressure $p_{\infty}$, i. e.,

$$
\left(p-p_{\infty}\right) / p_{\infty} \ll 1
$$

Consequently, the small disturbance theory is applicable to the flow fields in the incident and transmitted sides and the panel/acoustic interaction problem is described by (2.1) (2.6). The velocity potential $\Phi^{ \pm}(t, x, y, z)$ is governed by the simple wave equation,

$$
\left(C^{-2} \partial_{t t}^{2}-\partial_{x x}^{2}-\partial_{y y}^{2}-\partial_{z z}^{2}\right) \Phi^{ \pm}=0, \text { for } \quad \pm y>0
$$

and the acoustic pressure and velocity $\mathbf{v}^{ \pm}$are related to the potential by

$$
p^{ \pm}=-\rho_{\infty} \partial_{t} \Phi^{ \pm} \quad \text { and } \quad \mathbf{v}^{ \pm}=\nabla \Phi^{ \pm}
$$

In particular, we have $v=\partial_{y} \Phi$ where $v$ denotes the vertical velocity. The vertical displacement of the panel, $\eta(t, x, z)$, is governed by the system of partial differential equations (2.13). To produce the special forcing term, $\left(p^{(i)}+p^{(r)}\right)$, acting on the panel specified by (2.18) in Sec. 2.1, the incident potential should be

$$
\phi^{(i)}(t, x, y, z)=\frac{\epsilon}{2 \rho_{\infty} \omega C} \cos \left[\omega\left(t+\frac{y}{C}\right)\right] H\left(t+\frac{y}{C}\right) .
$$

Note that the incident wave $\phi^{(i)}$ is a solution of (2.22) in the whole space. When the panel is rigid, the incident wave is reflected by the rigid $(x, z)$ plane and the reflected wave is,

$$
\phi^{(r)}(t, x, y, z)=\phi^{(i)}(t, x,-y, z) \quad \text { for } \quad y \geq 0 .
$$


On the side $y<0$, the flow-field remains at rest because there is no transmitted wave.

For a flexible panel, the panel oscillation excited by the pressure difference across the panel $-2 p^{(i)}\left(t, x, 0^{+}, z\right)$ induces a scattered wave $\phi^{(s)}$ on the incident side and a transmitted wave $\phi^{(t)}$. Thus we write

$$
\Phi=\phi^{(i)}+\phi^{(r)}+\phi^{(s)}, \quad y>0
$$

and

$$
\Phi=\phi^{(t)}, \quad y<0
$$

Note that $\phi^{(s)}$ and $\phi^{(t)}$ are governed by the simple wave equation (2.22) in $y>0$ and $y<0$ respectively. The kinematic conditions on the $(x, z)$ plane, $(2.20)$ and $(2.23)$, become

$$
\partial_{y} \phi^{(s)}\left(t, x, 0^{+}, z\right)=\partial_{t} \eta(t, x, z) \quad \text { and } \quad \partial_{y} \phi^{(t)}\left(t, x, 0^{-}, z\right)=\partial_{t} \eta(t, x, z)
$$

where $\eta$ denotes the extension of the transverse displacement of the panel, $\mathcal{D}$, to the $(x$, z) plane, i. e.,

$$
\eta=0, \quad(x, z) \notin \mathcal{D}
$$

If the incident wave front hits the panel at $t=0$, we can impose the homogeneous initial conditions on $\phi^{(s)}, \phi^{(t)}$ and $\eta$,

$$
\phi^{(s)}=0, \partial_{t} \phi^{(s)}=0, \phi^{(t)}=0, \partial_{t} \phi^{(t)}=0 \quad \text { and } \quad \eta=0, \partial_{t} \eta=0 \quad \text { for } \quad t \leq 0
$$

Since the ambient fluid above and below the panel are the same, (2.27) implies that the velocity potential induced by the panel oscillation has to be anti-symmetric in $y$, i. e.,

$$
\phi^{(t)}(t, x, y, z)=-\phi^{(s)}(t, x,-y, z), \quad y<0
$$

Then the pressure difference across can be written as

$$
\Delta p=2 \rho_{\infty}\left[\partial_{t} \phi^{(i)}\left(t, x, 0^{+}, z\right)+\partial_{t} \phi^{(s)}\left(t, x, 0^{+}, z\right)\right], \quad(x, z) \in \mathcal{D}
$$


The derivation of the system of equations, (2.21) to (2.31), completes the formulation of the structural/acoustic interaction problem using linear theory for the acoustic fields. The panel oscillation, which can be nonlinear is governed by the system of equations, (2.13) (2.17).

The velocity potential $\phi^{(s)}$ induced by the panel oscillation $\eta$ is governed by the wave equation (2.22), the initial conditions (2.29) and the boundary condition (2.27). The solution is given by the Kirchhoff formula,

$$
\phi^{(s)}(t, x, y>0, z)=-\frac{1}{2 \pi} \iint_{\mathcal{G}} \frac{\left\{\eta_{t}\left(t, x^{\prime}, z^{\prime}\right)\right\}}{R} d x^{\prime} d z^{\prime},
$$

where $R=\left[\left(x-x^{\prime}\right)^{2}+y^{2}+\left(z-z^{\prime}\right)^{2}\right]^{1 / 2}$ denotes the distance from a point $(x, y>0, z)$ to a source at $\left(x^{\prime}, 0, z^{\prime}\right)$ and $\{\cdot\}$ denotes the retarded value of, i. e.,

$$
\left\{\eta_{t}\left(t, x^{\prime}, 0, z^{\prime}\right)\right\}=\eta_{t}\left(t-\frac{R}{C}, x^{\prime}, 0, z^{\prime}\right) .
$$

The domain of dependence of $\phi^{(s)}(t, x, y, z)$ is the circular disc $\mathcal{H}$ in the $\left(x^{\prime}, z^{\prime}\right)$ plane, $\mathrm{i}$. e.,

$$
\mathcal{H} \quad \mid R \leq C t \quad \text { or } \quad r^{2}=\left(x^{\prime}-x\right)^{2}+\left(z^{\prime}-z\right)^{2} \leq C^{2} t^{2}-y^{2} .
$$

The domain of integration in (2.32) is the intersection of $\mathcal{H}$ and the panel, i. e.,

$$
\mathcal{G}=\mathcal{H} \cap \mathcal{D}
$$

Now we introduce the polar coordinates, $r, \theta$, centered at $(x, z)$, i. e.,

$$
x^{\prime}-x=r \cos \theta \quad \text { and } \quad z^{\prime}-z=r \sin \theta
$$

and (2.32) becomes,

$$
\phi^{(s)}(t, x, y>0, z)=-\frac{1}{2 \pi} \int\left[\int_{\mathfrak{G}} d \theta g_{t}\left(t-\frac{R}{C}, r, \theta\right)\right] \frac{r d r}{R}
$$

with

$$
g(t, r, \theta)=\eta(t, x+r \cos \theta, z+r \sin \theta) .
$$


Here $R=\left(r^{2}+y^{2}\right)^{1 / 2}$ represents the slant height of a circular cone with vertex $\mathrm{P}(x, y, z)$, a vertical axis and a base circle of radius $r$ in the $(x, z)$ plane. On account of $(2.28)$, we can extend the domain of integration to $\mathcal{H}$ and rewrite (2.37) as an iterated integral,

$$
\phi^{(s)}(t, x, y>0, z)=-\frac{1}{2 \pi} \int_{0}^{C t}\left[\int_{0}^{2 \pi} d \theta g_{t}\left(t-\frac{R}{C}, r, \theta\right)\right] \frac{r d r}{R}
$$

The integral in $\theta$ represents the contribution of the sources on a base circle of radius $r$.

In (2.31), we need to relate the unknown, $\phi_{t}^{(s)}\left(x, 0^{+}, z\right)$ on the panel to $\eta(t, x, y)$ for $(x, z) \in \mathcal{D}$. This is obtained by differentiating the above equation with respect to $t$ and using (2.28). This results in replacing $\phi^{(s)}$ on the left-hand-side of (2.39) and $g_{t}$ in the integrand by $\phi_{t}^{(s)}$ and $g_{t t}$, respectively.

As $y \rightarrow 0^{+}, R \rightarrow r$ and the circular disc $\mathcal{H}$ is bounded by the sonic circle $r=C t$. Equation (2.39) yields

$$
\phi_{t}^{(s)}\left(t, x, 0^{+}, z\right)=-\frac{1}{2 \pi} \int_{0}^{2 \pi} \int_{0}^{C t} g_{t t}\left(t-\frac{r}{C}, r, \theta\right) d r d \theta .
$$

Note that in this form we remove the kernel $1 / R$ in (2.32), which becomes singular as $y \rightarrow 0$ and $r \rightarrow 0$ and shows that the area of the domain of integration is bounded above by $2 \pi C t$. By using (2.31) and (2.40), (2.13) becomes a system of integro-differrential equations for the panel oscillation, $\eta$. The initial and boundary conditions for $\eta$ are (2.16) and (2.17). Thus we complete the formulation of the decoupled model. The numerical solution of this system will be described in Sec. 3 .

\subsection{Application to Noise Transmission in a Supersonic Flight at High Altitude}

The analysis reported in Sec. 2.2 is based on two assumptions

(i) The flow-fields above and below the panel have the same ambient condition, i. e., $\rho_{\infty}^{+}=\rho_{\infty}^{-}, p_{\infty}^{+}=p_{\infty}^{-}$and $\mathbf{v}_{\infty}^{ \pm}=0$.

(ii) The panel is planar and not prestressed, i. e., under zero tension. 
The analysis can be employed to estimate the noise transmitted into the fuselage flying at low speed and a relatively low level, where the static pressure remains nearly equal to one atmosphere. Assumption (i) is valid when terms of the order of the flight Mach number $M$ are omitted. Assumption (ii) is valid since the size of the panel $L$ is much smaller than the radius of the fuselage, $R_{f}$. There is no hoop stress when $p_{\infty}^{+}=p_{\infty}^{-}$under assumption (i). In practice, there is a pressure difference $\left(p_{\infty}^{-}-p_{\infty}^{+}\right)$, even for a low Mach number flight.

For an airplane cruising at subsonic or supersonic speed, it is necessary to account for the differences in densities and in pressures inside and outside the fuselage and the moving media outside and to include the contribution of the hoop stress to the panel oscillation. The formulation of the decoupled models for structural acoustic interactions in a moving media at subsonic and supersonic speeds is carried out using the linear theory for the flowfield. The system of integro-differential equations for the panel oscillation is then reduced to a system of differential equations when the acoustic wave length is much smaller than the surface wave length [11].

In this subsection, we show that with minor modifications the above analysis becomes applicable to a supersonic airplane cruising at high altitude. We make use of the fact that the cabin pressure, $p_{\infty}^{-}$, and temperature and hence the density are maintained near the ambient level while the outside pressure $p_{\infty}^{+}$and density $\rho_{\infty}^{+}$at the flight altitude $H$ are much lower. The density ratio, $\rho_{\infty}^{+} / \rho_{\infty}^{-}$is about $1 / 10$ or $1 / 20$ when $H=60,000$ or 80,000 $\mathrm{ft}$. The same is true for the pressure ratio while the absolute temperature ratio and hence the ratio of the speed of sound remains $O(1)$, i. e.,

$$
\mu=\rho_{\infty}^{+} / \rho_{\infty}^{-} \ll 1, \quad p_{\infty}^{+} / p_{\infty}^{-}=O(\mu) \text { and } C^{+} / C^{-}=O(1)
$$

Thus, the density ratio $\mu$ serves as a small parameter in the following modification of the analysis in Sec. 2.2 .

On the transmitted side, $y<0$, the governing equations, (2.13) (2.23), (2.26b), (2.27) and (2.29), remain valid when the superscript "-" is added to $p_{\infty}$ and $C$. On the incident side, $y>0$, equations $(2.25),(2.26 \mathrm{a})$, and (2.29) remain the same while $(2.22),(2.23)$ 
and (2.27) have to be modified to include the convection terms. Consequently, (2.30) and (2.31) will be modified. In particular, we note the acoustic pressure relationship (2.23) in the transmitted side,

$$
p^{-}-p_{\infty}^{-}=p^{(t)}=-\rho_{\infty}^{-} \partial_{t} \phi^{(t)}, \quad y<0
$$

and that on the incident side,

$$
p^{+}-p_{\infty}^{+}=p^{(i)}+p^{(r)}+p^{(s)}=-\mu \rho_{\infty}^{-}\left[\partial_{t}+M C^{+} \partial_{x}\right] \Phi, \quad y>0
$$

Using (2.26a) and (2.43), we have

$$
p^{(s)}=-\mu \rho_{\infty}^{-}\left[\partial_{t}+M C^{+} \partial_{x}\right] \phi^{(s)} \quad y>0,
$$

Likewise, the kinematic condition on the $y=0^{+}$is modified and (2.27) becomes,

$$
\partial_{y} \phi^{(s)}\left(t, x, 0^{+}, z\right)=\left[\partial_{t}+M C^{+} \partial_{x}\right] \eta(t, x, z) \quad \text { and } \quad \partial_{y} \phi^{(t)}\left(t, x, 0^{-}, z\right)=\partial_{t} \eta(t, x, z)
$$

With the convection term at most of the order of the unsteady term, or one order smaller under the short acoustic wave approximation, we deduce from (2.45)

$$
\partial_{y} \phi^{(t)}\left(t, x, 0^{-}, z\right)=O\left(\partial_{y} \phi^{(s)}\left(t, x, 0^{+}, z\right)\right)
$$

and then from (2.44)

$$
p^{(s)}\left(t, x, 0^{+}, z\right)=O\left(\mu p^{(t)}\left(t, x, 0^{-}, z\right)\right)
$$

By using (2.41) and (2.44), the load on the panel, which is the pressure difference, becomes

$$
\begin{aligned}
\Delta p & =\left(p_{\infty}^{-}-p_{\infty}^{+}\right)+\left[-2 p^{(i)}+\left(p^{(t)}-p^{(s)}\right)\right] H(t) \\
& =p_{\infty}^{-}[1-O(\mu)]+\left[-2 p^{(i)}+p^{(t)}(1+O(\mu)] H(t) .\right.
\end{aligned}
$$

The constant load $\left(p_{\infty}^{-}-p_{\infty}^{+}\right) \approx p_{\infty}^{-}$is present for all $t$. The unsteady load due to the acoustic waves is present only for $t>0$ and is much smaller then $p_{\infty}^{-}$. The constant load 
produces a static deflection $\eta_{0}(x, z)$ which does not generate far-field sound. The unsteady load excites panel oscillation $\eta^{\prime}(t, x, z)$ and generates the far-field sound. Therefore, we separate the static panel deflection from the unsteady oscillation,

$$
\eta=\eta_{0}(x, z)+\eta^{\prime}(t, x, z) .
$$

Note that the panel oscillation, $\eta$, is coupled with only the acoustic field (2.48) in the transmitted side, since $p^{(i)}$ is prescribed and $p^{(s)}$ is $O(\mu)$ related to $p^{(t)}$. This is equivalent to say that in a high altitude flight the acoustic damping in the flow-field outside of the fuselage is of the order of the density ratio $\mu=\rho_{\infty}^{+} / \rho_{\infty}^{-}$relative to that inside and hence is neglegible.

Since the fluid is at rest inside the fuselage, the transmitted wave obeys the simple wave equation (2.22). We can make use of the analysis in Sec. 2.2, in particular, (2.30) and $(2.40)$, to relate the on surface transmitted pressure to the panel oscillation,

$$
p^{(t)}\left(t, x, 0^{-}, z\right)=-\rho_{\infty}^{-} \partial_{t} \phi^{(t)}=-\frac{\rho_{\infty}^{-}}{2 \pi} \int_{0}^{2 \pi} \int_{0}^{C^{-} t} g_{t t}\left(t-\frac{r}{C^{-}}, r, \theta\right) d r d \theta
$$

Thus we have a closed system for the panel oscillation, $\eta^{\prime}$, from which we can then compute the transmitted and scattered acoustic fields.

\section{Numerical Methods}

For the fully coupled model, the nonlinear Euler equations, (2.7), are solved using an explicit finite difference scheme. The scheme, which is a generalization of MacCormack's scheme obtained by Gottlieb and Turkel [12], is fourth-order accurate in space and secondorder accurate in time. Further details on the implementation of the scheme can be found in Frendi et al. [1,2]. The physical boundary of the computational domain (the bottom boundary, see Fig. 1) is composed of a flexible panel clamped between rigid plates. Over the rigid plates, the vertical velocity is zero $(v=0)$ and the surface temperature is $T_{w}$, which in this paper is the same as the fluid temperature, $T_{\infty}$. The $x$ and $z$ components of the velocity ( $u$ and $w$, respectively) are obtained through linear extrapolation from the 
interior of the computational domain. The pressure boundary condition is obtained using the normal momentum equation by simply imposing the normal gradient of the sum of pressure and vertical momentum flux to be zero, i. e., $\partial_{y}\left(p+\rho v^{2}\right)=0$. Over the flexible panel, the vertical velocity is set to be equal to that of the panel and the temperature is $T_{w}$. The $x$ and $z$ velocity components and the pressure are extrapolated from the interior of the computational domain. The appropriate boundary conditions on the computational domain are derived using the method of characteristics [13]. One should mention that the characteristic boundary conditions and the extrapolation are only first order accurate, while the interior scheme is fourth order. These boundaries are believed to be a source for numerical error in the fully coupled model. The dimensions of the computational domain are, $61 \mathrm{~cm}, 3.05 \mathrm{~m}$ and $40.64 \mathrm{~cm}$ in the $x, y$ and $z$ directions, respectively. The number of computational points used are, 121,241 , and 81 in the respective directions.

The nonlinear plate equations, (2.13), are solved using a finite element method developed by Robinson [14]. The panel is $30.5 \mathrm{~cm}$ long, $20.32 \mathrm{~cm}$ wide and $0.102 \mathrm{~cm}$ thick, and the number of elements used are 6 and 8 respectively.

Since the grid used for solving the plate equations is rectangular, it is easlier to evaluate the integral in (2.40) in cartesian coordinates. In this case there is a singularity at each computational point corresponding to $r=0$. In order to overcome this difficulty, a Taylor series expansion up to the second order is used to calculate the contribution of the singular point. The contribution of the various points on the panel that lie within the sonic circle $(R \leq C t)$ is calculated by first integrating in $x$ using a combination of Simpson and trapezoidal rules. The result is then integrated in $z$ using Simpson's rule of integration. Because of the presence of the retarded time in the integral, the vertical velocity of the plate $\left(\eta_{t}\right)$ is stored at each point for several time-steps. The evaluation of the double integral can use a much larger time step than the $\Delta t$ for the integration of the differential equations, yet having the same degree of accuracy. For a plate of given dimensions $(L, W)$, and for a fixed time-step $\Delta t$, the maximum number of time-steps to be stored is $N=\sqrt{L^{2}+W^{2}} /(C \Delta t)$. The number $N$ has to be changed when calculating the radiated pressure away from the plate. The number $N$ is related to the radius of the 
largest sonic circle in the domain that contains the flexible panel. For the cases presented in the next section $N=1503$ for a time-step $\Delta t=4 \times 10^{-6}$ sec.

\section{Results and Discussion}

The numerical schemes for the two models presented in Sec. 3 are used to predict the vibration of a flexible panel and the resulting acoustic radiation. The panel is forced to vibrate by harmonic plane acoustic waves at normal incidence. The frequency of the source is $751 \mathrm{~Hz}$, which corresponds to a natural frequency of the panel. Two different amplitudes of the incident waves are used. The properties of the panel, which are considered to be uniform, are: density $\rho_{p}=4450.15 \mathrm{Kg} / \mathrm{m}^{3}$, modulus of elasticity $E=1.10316 \times 10^{11} \mathrm{~N} / \mathrm{m}^{2}$, Poisson ratio $\nu=0.33$ and a damping ratio of 0.01 . The acoustic fluid properties are: temperature $T_{\infty}=288.33{ }^{\circ} \mathrm{K}$, density $\rho_{\infty}=1.23 \mathrm{Kg} / \mathrm{m}^{3}$, pressure $p_{\infty}=1.013 \times 10^{5} \mathrm{~N} / \mathrm{m}^{2}$ and sound speed $c_{\infty}=340 \mathrm{~m} / \mathrm{sec}$. The specific heat at constant volume is $c_{v}=1.004$ $\mathrm{KJ} /\left(\mathrm{Kg}{ }^{\circ} \mathrm{K}\right)$, the ratio of specific heats is $\gamma=c_{p} / c_{v}=1.4$. In the-far field, the fluid is at rest. The variables plotted on figures $2-5$ are nondimensional. The reference quantities are given by

$$
\begin{gathered}
(x, y, z, \eta)_{r e f}=l_{\text {ref }}, \quad t_{r e f}=\frac{l_{\text {ref }}}{C}, \quad \text { and } \quad T_{r e f}=\frac{C^{2}}{c_{v}} \\
\rho_{\text {ref }}=\rho_{\infty}, \quad\left(u, v, w, \frac{\partial \eta}{\partial t}\right)_{r e f}=C, \quad \text { and } \quad(p, e)_{r e f}=\rho_{\infty} C^{2} .
\end{gathered}
$$

where the reference length is $l_{\text {ref }}=0.3048 \mathrm{~m}$.

Figure 1 shows the configuration of the computational domain, a rectangular box with the lower side composed of a flexible panel clamped between rigid plates. For a low excitation amplitude, $100 \mathrm{~dB}$ or $5 \times 10^{-4}$ atm., Fig. 2 shows that the panel response is linear. In this case, both models predict the same panel response as shown by the figure. This result is expected since the incident wave is extremely weak. Figure $3 \mathrm{a}-\mathrm{b}$ show the time histories of the radiated near- and far-field pressures, $2.54 \mathrm{~cm}$ and $1.524 \mathrm{~m}$, or $L / 12$ and $5 L$, away from the panel center respectively. The radiated pressure predicted by the linear theory (2.32) is in excellent agreement with that predicted by the Euler equations $(2.7)$. 
When the level of the excitation is increased to $160 \mathrm{~dB}$, or $0.01 \mathrm{~atm}$, the response of the panel becomes nonlinear as shown by Fig. 4. The time history of the panel center displacement shows a non-periodic behaviour characteristic of nonlinear response. The figure also shows that even when the panel oscillation is nonlinear, the predictions of the two models are in reasonably good agreement, because the pressure variation is still much smaller than $p_{\infty}=1 \mathrm{~atm}$. The radiated near- and far-field pressure time histories are shown on Fig. 5a-b. In the near-field, $L / 12$, the predictions of the two models are in reasonably good agreement and the maximum pressure variation is of the order of 0.02 atm. In the far-field, $5 L$, as shown in Fig. 5b, the maximum pressure variation is of the order of $10^{-4}$ atm. and is reduced by a factor of 100 from that in the near-field. In Fig. $5 b$, the difference between the "decoupled model" and the "fully coupled model" can be obseved. But this difference is of the order of $10^{-3}$ times the pressure variation in the near-field and is within the accuracy of the numerical solution.

From a computational view point, it is important to compare the performance of the two models based on the $\mathrm{CPU}$ time required by each calculation. In the linear vibration regime, the "fully coupled model" used 36000 seconds of CPU time on a Cray-ymp to advance the calculation by 10000 time-steps, whereas the "decoupled model" used only 1000 seconds for the same calculation. In the nonlinear vibration regime, grid refinements were needed to resolve the large gradients both on the panel and in the radiation field. Therefore, in order to advance the calculation by 10000 time-steps, 72000 seconds were used by the "fully coupled model" while the "decoupled model" used only 1200 seconds.

\section{Conclusions}

An efficient model for coupling the vibration of a panel to the on surface acoustic radiation was derived. The model uncouples the panel vibrations from the acoustic wave propagation problem. The results showed that this model, referred to as "decoupled model", accurately predicts the panel response and acoustic radiation in the linear and nonlinear vibration regimes so long as the pressure variation in the flow-field remains much smaller than the ambient pressure. For the cases studied in this paper, the computational cost of 
the numerical integration of this model is 36 times cheaper in the linear regime and 60 times cheaper in the nonlinear regime than the cost of the "fully coupled model".

\section{Acknowledgements}

The first author acknowledges the support of NASA Langley Research Center under contract NAS1-19700. Partial support was also provided by NASA Langley to the third author under contracts NAS1-18605 and NAS1-19480 while in residence at ICASE.

\section{References}

[1] A. Frendi and J. Robinson, "On the Effect of Acoustic Coupling on Random and Harmonic Plate Vibrations", NASA-TM-107740 (1993).

[2] A. Frendi, L. Maestrello and A. Bayliss, "Coupling Between Plate Vibration and Acoustic Radiation", ICASE Report 92-67 (1992).

[3] L. Maestrello, A. Frendi and D. E. Brown, "Nonlinear Vibration and Radiation from a Panel with Transition to Chaos induced by acoustic waves", AIAA Journal 30, No. 11, pp. 2632-2638 (1992).

[4] P. M. Morse and K. U. Ungard, Theoretical Acoustics, McGraw-Hill, New York, (1968).

[5] G. A. Kriegsmann and C. Morawetz, "Solving the Helmhotz Equation for Exterior Problems with Variable Index of Refraction: I", SIAM J. Sci. Stat. Comput. 1, pp. 371-385 (1980).

[6] A. Bayliss and E. Turkel, "Radiation Boundary Conditions for Wave-Like Equations", Comm. pure Appl. Math. 23, pp. 707-725 (1980).

[7] L. Ting and M. J. Miksis, "Exact Boundary Conditions for Scattering Problems", $J$. Ascout. Soc. Amer. 80, pp. 1825-1827 (1986).

[8] M. J. Miksis and L. Ting, "Scattering of an Incident Wave from an Interface Separating two Fluids", Wave Motion 11, pp. 545-557 (1989).

[9] M. J. Míksis and L. Ting, "Scattering of Acoustic Wave with a Flexible Wall", Northwestern University Report 9130, June 1992. 
[10] C. Y. Chia, "Nonlinear Analysis of Plates", McGraw-Hill, New York, pp. 1-50 (1990).

[11] L. Ting, "On Surface conditions for Structural Acoustic Interactions in Moving Media", to be presented in A Workshop on Perturbation Methods in Physical Mathematics, Jume 23-26, 1993, Rensselaer Polytechnic Institute, Troy, NY.

[12] D. Gottlieb) and E. Turkel, "Dissipative Two-Four Methods for Time Dependent Problems", Math. Comp. 30, pp. 703-723 (1976).

[13] S. S. Abarbanel, W. S. Don, D. Gottlieb, D. H. Rudy, and J. C. Townsend, "Secondary Frequencies in the Wake of a Circular Cylinder with Vortex Shedding", ICASE Report 90-16 (1990).

[14] J. H. Robinson, "Finite Element Formulation and Numerical Simulation of the Ranlom Response of Composite Plate", Master's Thesis, Mechanical Engineering Department, Old Dominion University, Norfolk, VA, (1990). 


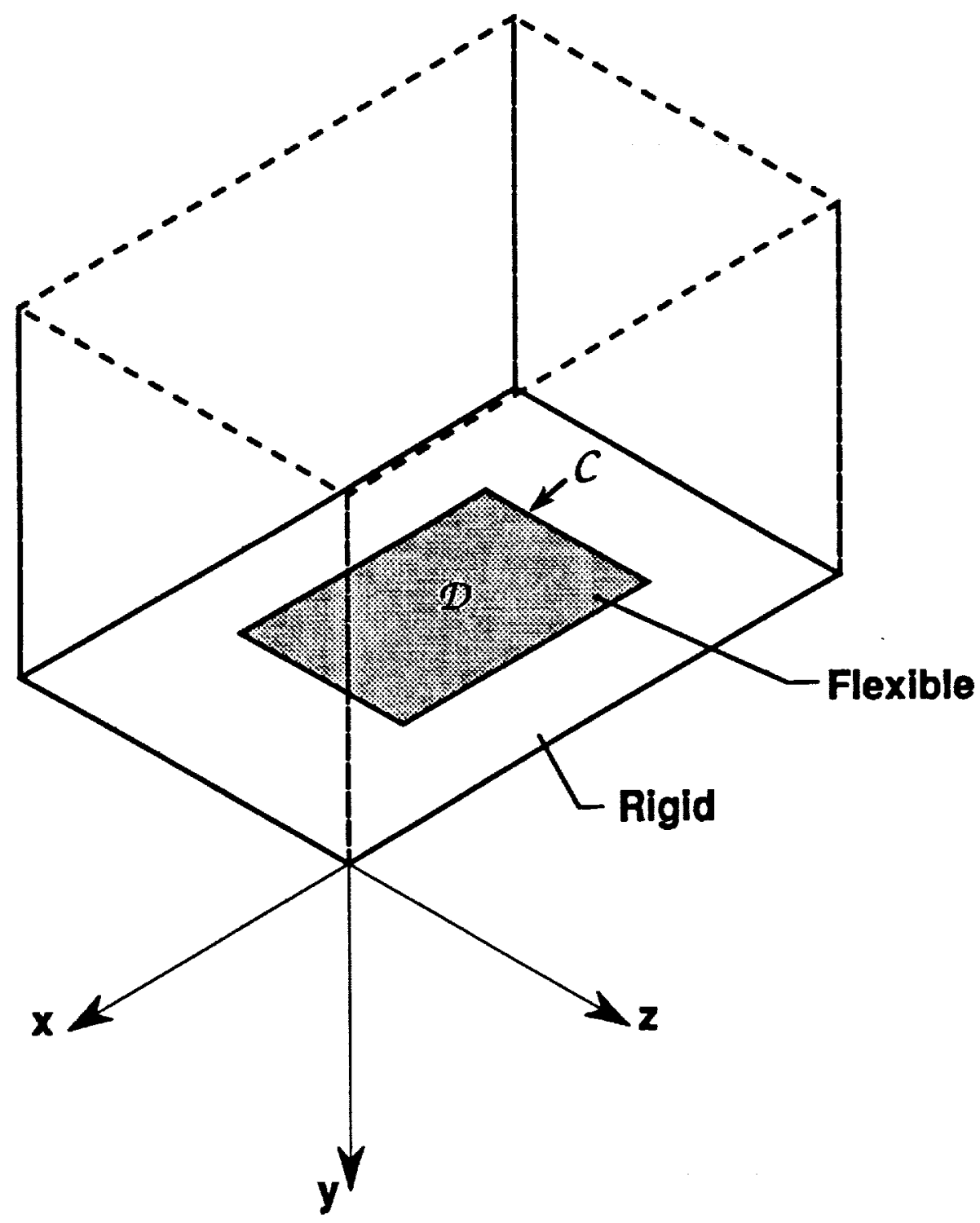

Figure 1: Computational Domain 


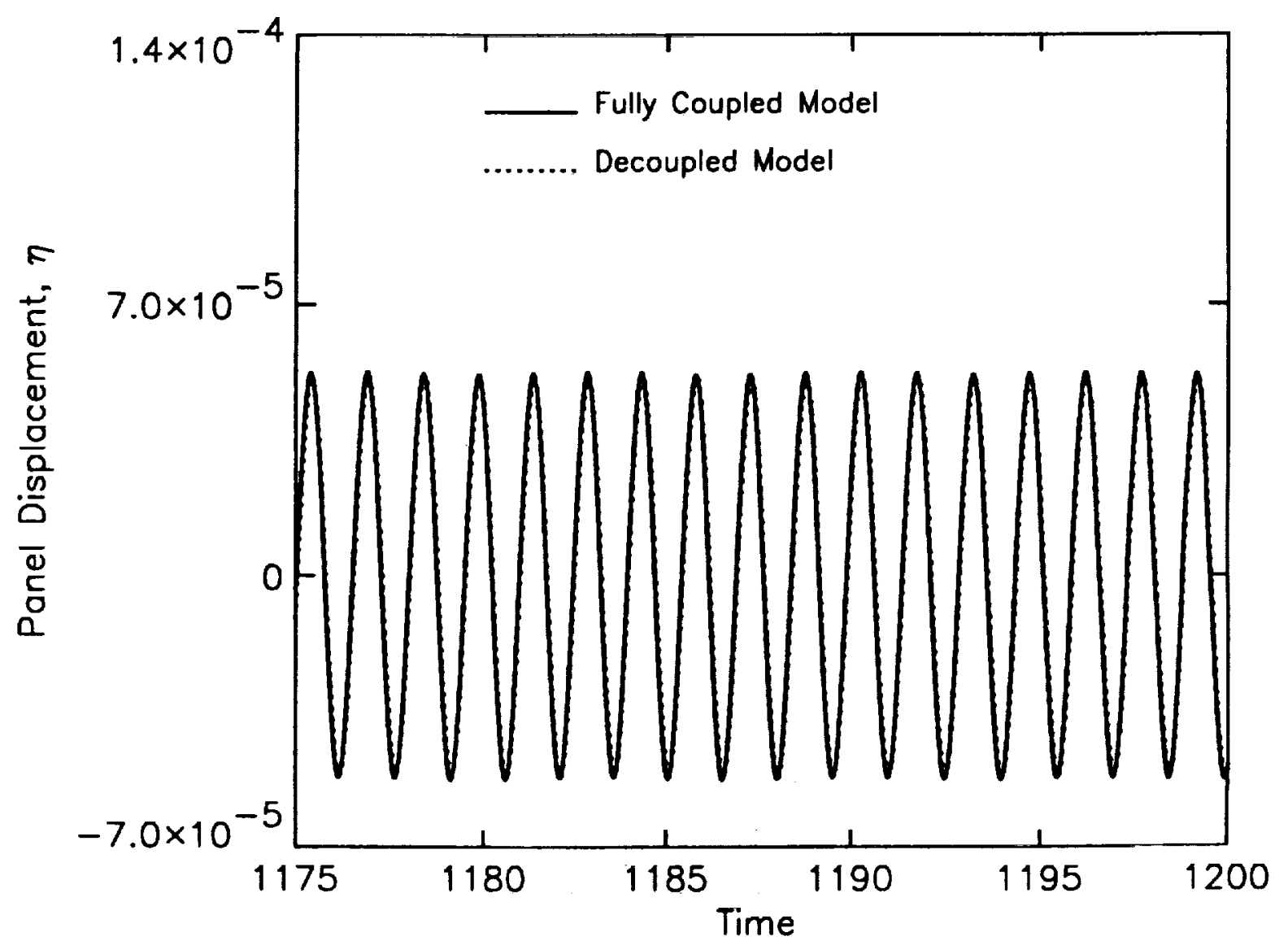

Figure 2: Time history of the displacement of the panel center for an excitation amplitude of $100 \mathrm{~dB}$ (linear). 

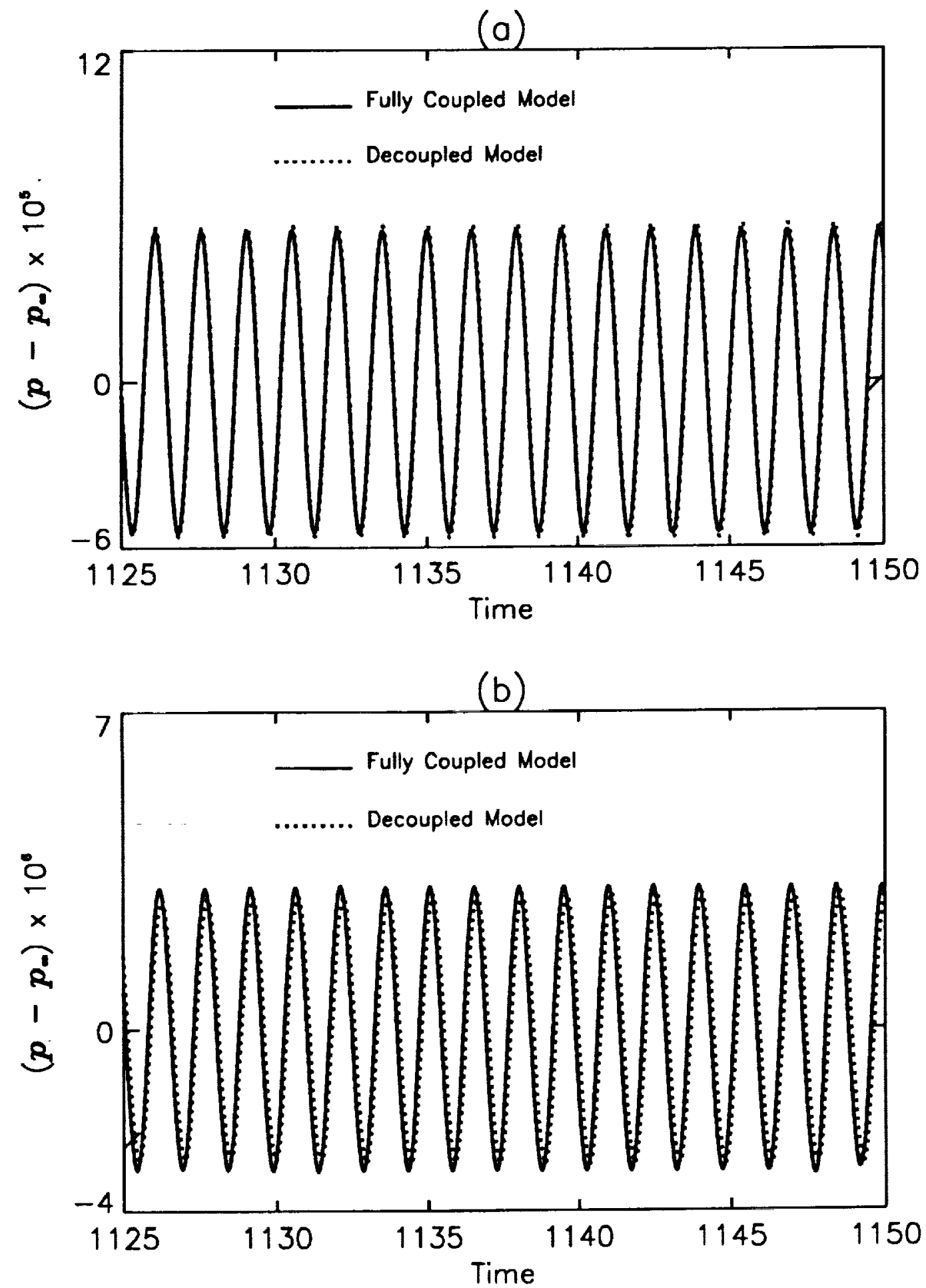

Figure 3: Time history of the radiated pressure along a line perpendicular to the panel center. (a) 1 inch away from the panel, (b) 60 inches away from the panel. 


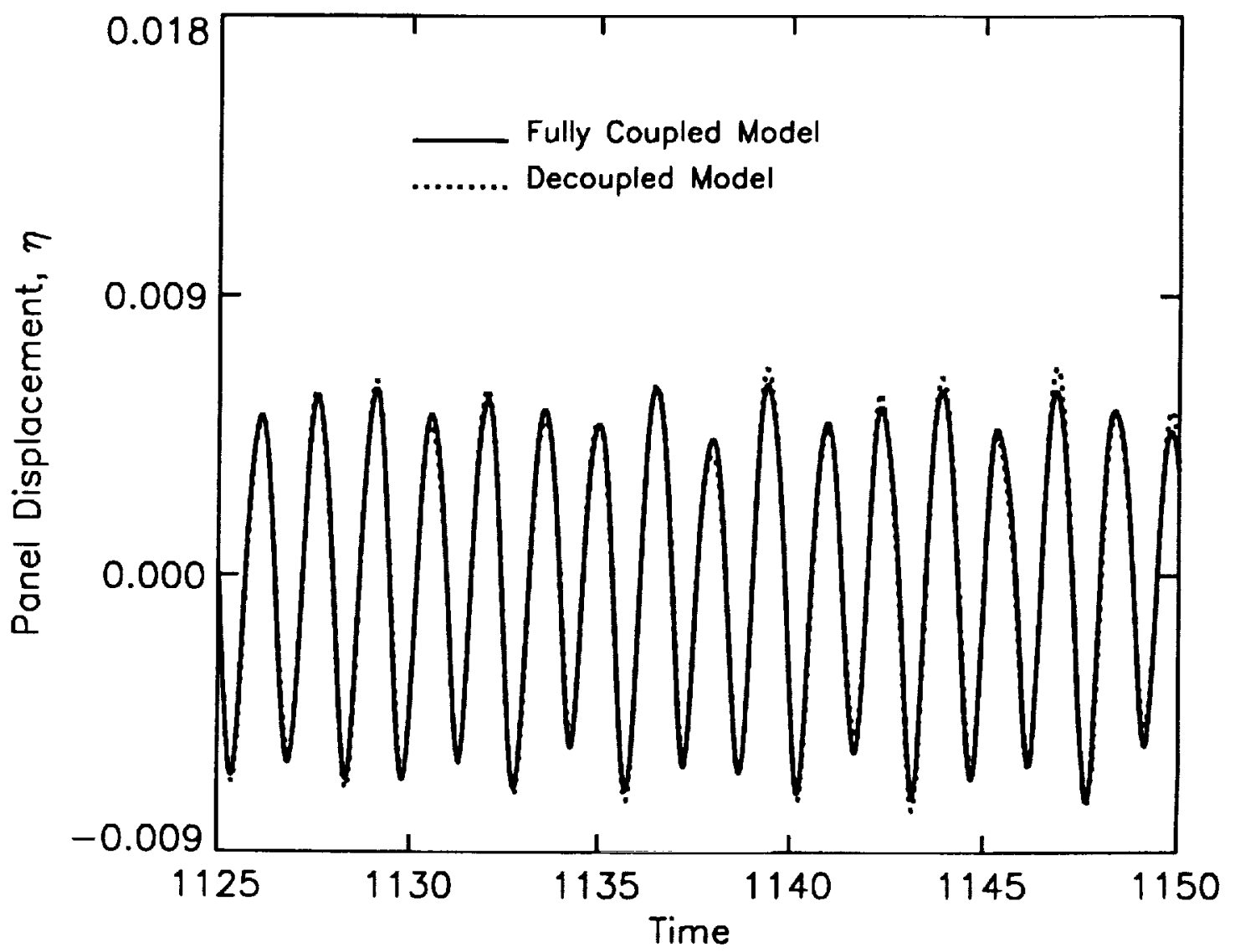

Figure 4: Time history of the displacement of the panel center for an excitation amplitude of $160 \mathrm{~dB}$ (nonlinear). 


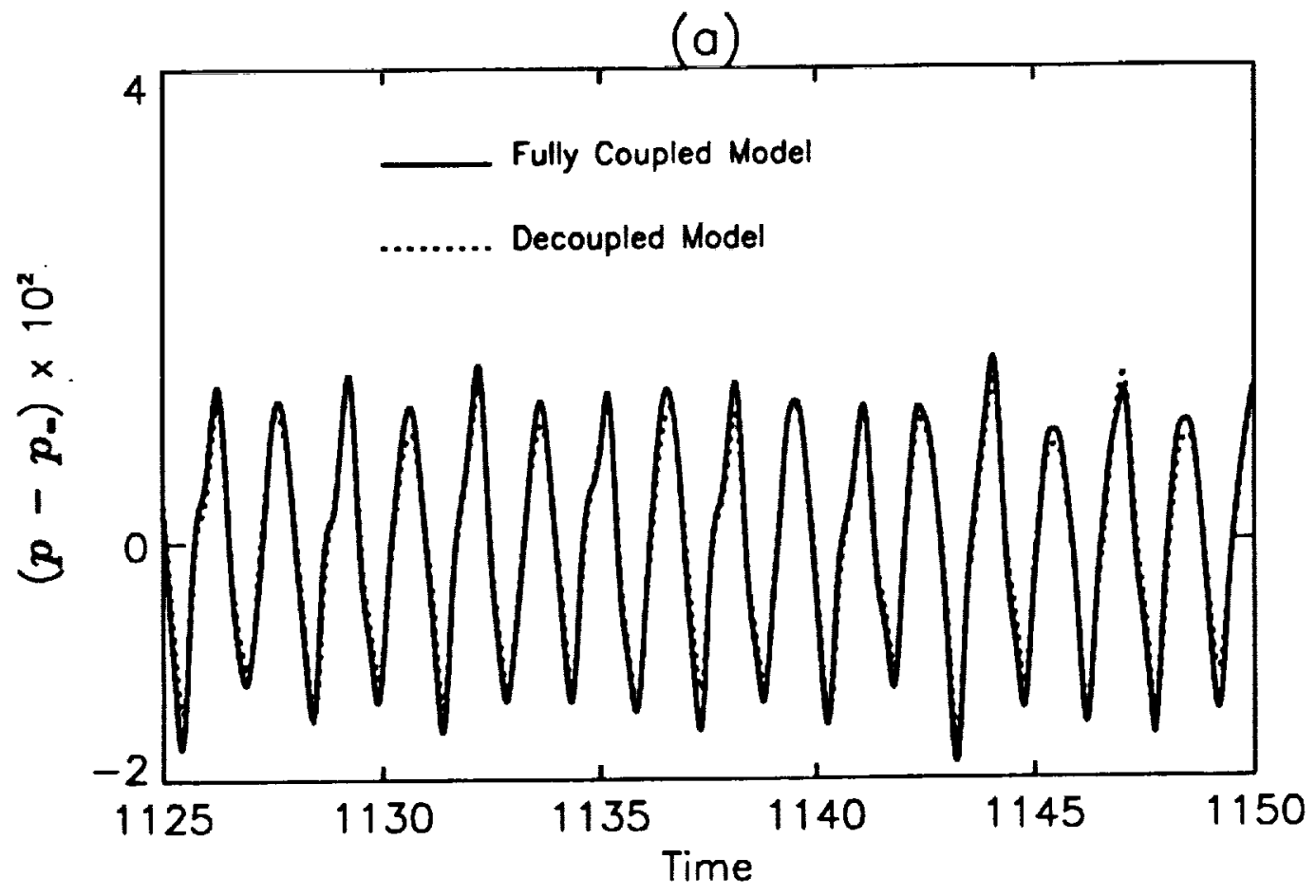

(b)

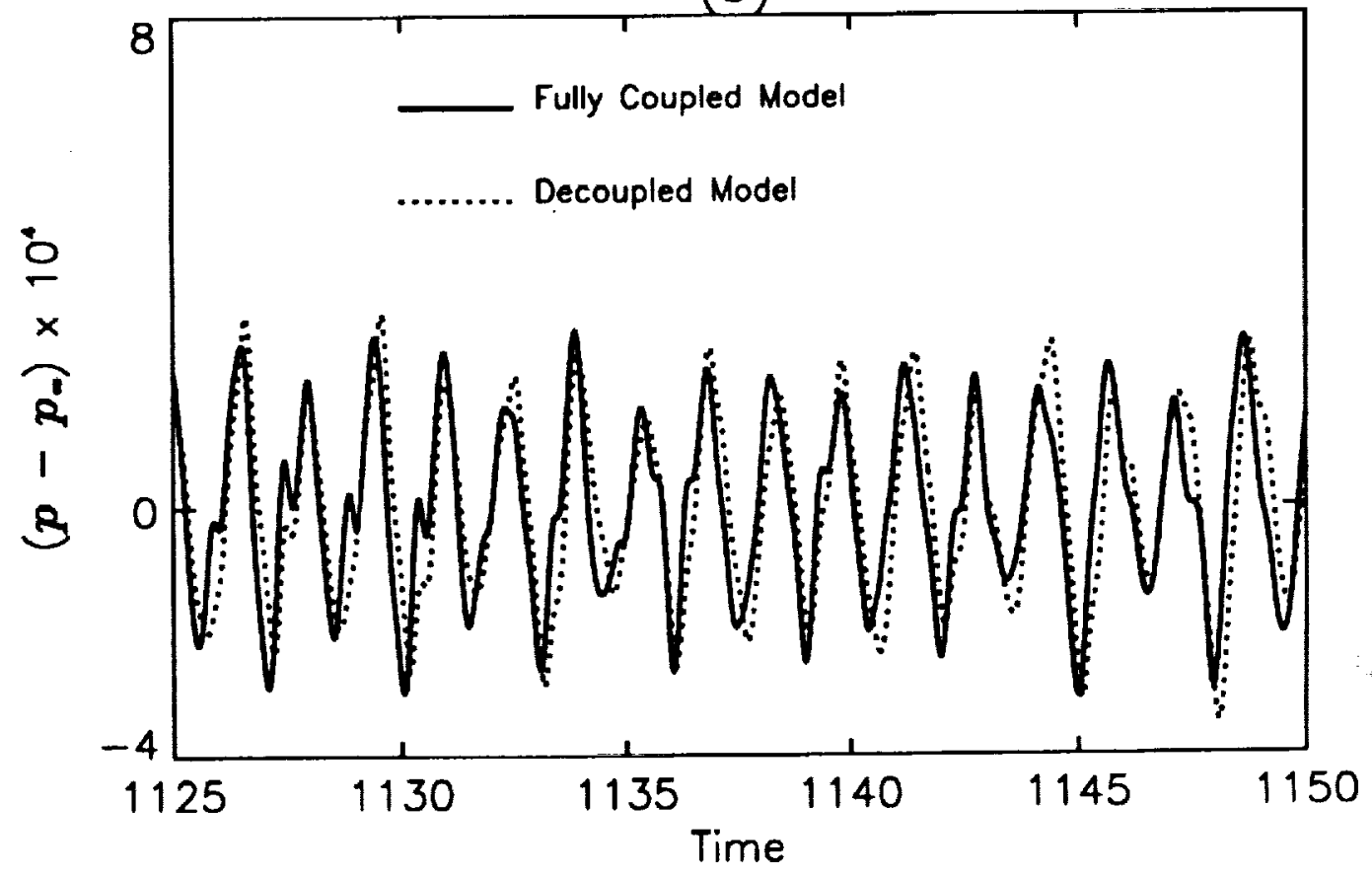

Figure 5: Time history of the radiated pressure along a line perpendicular to the panel center. (a) 1 inch away from the panel, (b) 60 inches away from the panel.

*U.S. GOVFRNMENT PRINTING OFFICE: 1993-728-150/60050 


\section{REPORT DOCUMENTATION PAGE}

Public reporting burden for this collection of information is estimated to average 1 hour per response, including the time for reviewing imstructions, searching existing data sources gathering and maintaining the dats needed, and completing and reviewing the coliection of information. Send comments regarding this burden estions and heports. 1215 jefferson

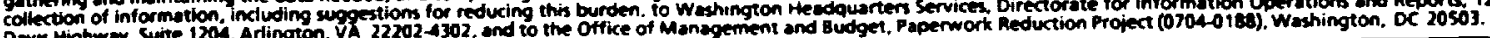

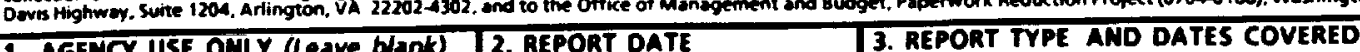

\begin{tabular}{|l|l|l} 
1. AGENCY USE ONLY (Leave blank) & $\begin{array}{l}\text { 2. REPORT DATE } \\
\text { Apri1 } 1993\end{array}$ & $\begin{array}{r}\text { 3. REPORT TYPE AND DATES } \\
\text { Contractor Report }\end{array}$
\end{tabular}

4. TILE AND SUBTTLE

AN EFFICIENT MODEL FOR COUPLING STRUCTURAL VIBRATION WITH ACOUSTIC RADIATION

\section{AUTHOR(S)}

Abdelkader Frendi, Lucio Maestrello, and Lu Ting

\section{PERFORMING ORGANIZATION NAME(S) AND ADDRESS(ES)}

Institute for Computer Applications in Sclence

and Engineering

Mall Stop 132C, NASA Langley Research Center

Hampton, VA 23681-0001

\section{SPONSORING/MONITORING AGENCY NAME(S) AND ADDRESS(ES)}

National Aeronautics and Space Administration

Langley Research Center

Hampton, VA 23681-0001

\section{FUNDWNG NUMBERS}

C NAS1-19480

C NAS1-18605

WU 505-90-52-01
8. PERFORMING ORGANIZATION REPORT NUMBER

ICASE Report No. 93-18

10. SPONSORING/MONITORING AGENCY REPORT NUMBER

NASA CR-191452

ICASE Report No. 93-18

\section{SUPPLEMENTARY NOTES}

Langley Technical Monitor: Michael F. Card

Final Report
To be submitted to Journal of Sound and Vibration

12.. DISTRIBUTION/AVALLABLITY STATEMENT

12b. DISTRIBUTION CODE

Unclassified - Unlimited

Subject Category $71,64,66$

13. ABSTRACT (Maximum 200 words)

We study the scattering of an incident wave by a flexible panel. The panel vibration is governed by the nonlinear plate equations while the loading on the panel, which is the pressure difference across the panel, depends on the reflected and transmitted waves. Two models are used to calculate this structural-acoustic interaction problem. One solves the three dimensional nonlinear Euler equations for the flow-fleld coupled with the plate equations (the fully coupled model). The second uses the linear wave equation for the acoustic fleld and expresses the load as a double integral involving the panel oscillation (the decoupled model). The panel oscillation governed by a system of integro-differential equations is solved numerically and the acoustic field is then defined by an explicit formula. Numerical results are obtained using the two models for linear and nonlinear panel vibrations. The predictions given by these two models are in good agreement but the computational time needed for the "fully coupled model" 1860 times longer than that for "the decoupled model".

\begin{tabular}{|c|c|c|c|}
\hline $\begin{array}{l}\text { 14. SUBJECT TERMS } \\
\text { coupling, acoustic r } \\
\text { nonlinear }\end{array}$ & dation, structural v & ration, linear, & $\begin{array}{l}\text { 15. NUMBER OF PAGES } \\
26 \\
\begin{array}{l}\text { 16. PRICE CODE } \\
\text { A0 } 3\end{array}\end{array}$ \\
\hline $\begin{array}{l}\text { 17. SECURTY QASSIFICATION } \\
\text { Of REPOAT } \\
\text { Unclasa1fled }\end{array}$ & $\begin{array}{l}\text { 18. SECURITY CLASSIFICATION } \\
\text { OF THIS PAGE } \\
\text { Unclass } 1 \text { fled }\end{array}$ & $\begin{array}{l}\text { 19. SECURITY CLASSIFICATION } \\
\text { OF ABSTRACT }\end{array}$ & 20. LIMITATION OF ABSTRACT \\
\hline
\end{tabular}


皇

$=$ 